\title{
OBSERYACIONES ECOLOGICAS DE LA ICTIOFAUNA DE UNA PRADERA DE THALASSIA EN LA BAHIA DE NENGUANGE (PARQUE NACIONAL TAYRONA, COLOMBIA)
}

ARTURO ACERO P.

Instituto de Investigaciones Marinas de Punta de Betín (INVEMAR), Santa Marta, Colombia

\section{SYNOPSIS}

In a turle-grass bed (Thalassia testedinum) next to a coral reef (Acropora spp.), 105 species of fish were observed, $42 \%$ of them belonged to the community of benthic residents, $45 \%$ to suprabenthic nomads and $13 \%$ were transients. The importance of Millepora complanata as shelter for the benthic residents is emphasized; it is thought that the distribution of the species observed is based upon trophic differences.

\section{Introduccion}

En los arrecifes coralinos los peces forman parte fundamental al constituirse en elementos claves de la red trófica, en tanto que las praderas de Thalassia testudinum Banks son muy importantes para la ictiofauna arrecifal caribeña (Ehrlich, 1975). En la costa Caribe de Colombia se destaca el Parque Nacional Tayrona, el cual está conformado por varias bahías en cuyas orillas orientales existen arrecifes coralinos. Este estudio se desarrolló en la región oriental de la Bahía de Nenguange, donde se han efectuado otros trabajos (Cayecedo, 1977). Se escogió una pradera de Thalassia delimitada al norte y al sur por formaciones rocosas y separada del interior de la bahía por arrecifes coralinos (Fig. 1). En la pradera se desarrollan formaciones coralinas que se encuentran dispersas en toda su extensión, de las cuales se escogieron para estudiar los peces relacionados con ellas las siguientes:

A: aguas someras y quietas, Millepora complanata Lamarck.

B: aguas someras y poco movidas, Acropora palmata (Lamarck).

C: aguas someras y poco movidas, A. cervicornis (Lamarck).

$\mathrm{D}$ : aguas someras y muy movidas, $A$. palmata.

$\mathrm{E}$ : aguas someras y quietas, $P$. porites (Pallas) muerto.

$\mathrm{F}$ : aguas menos someras y movidas, Siderastrea radians (Pallas) y M. complanata.

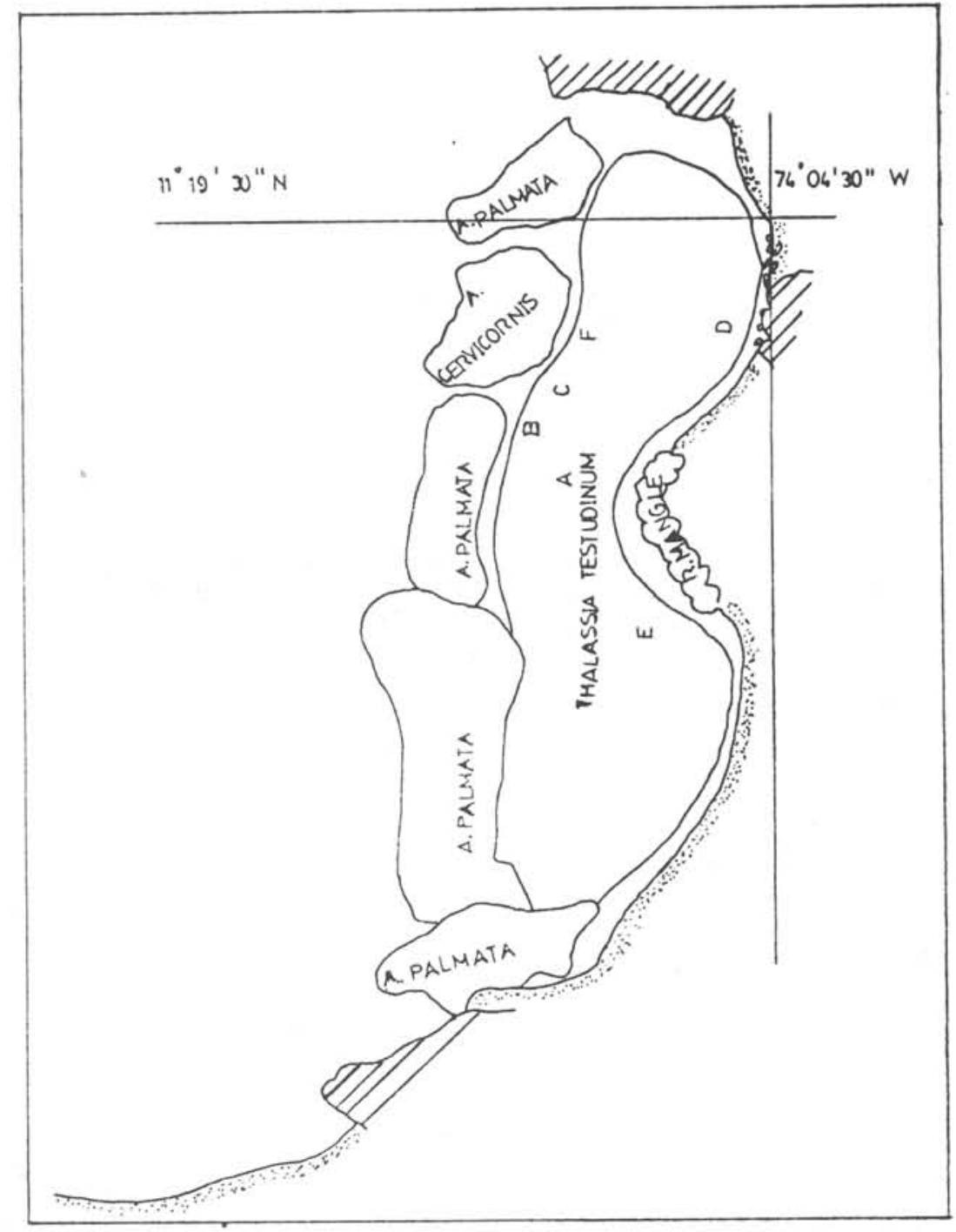

Fig. 1. Mapa de la zona estudiada. 
Para identuficar los peces fueron utilizadas las obras de Böhlke \& Chaplin (1968), Randall (1968) y Chaplin \& Scott (1972). se buceo con equipe convencional durante 130 horas entre $\mathrm{Fe}$ brero y Diciembre de 1976.

Relaciones por espacio

Las especies que habitan la pradera son ubicadas en tres comunidades, de acuerdo a sus retaciones con las tormaciones coralinas esparcidas en ella $y / 0$ con la pradera propriamente dicha, residentes bénticos, nómadas suprabénticos y visitantes de media aqua. definidas por Smith \& Tyler $(1972,1973)$. En las Tablas I, II y IIIIvemos las especies ubicadas en el grupo que les corresponde, quedando 44 especies como residentes, 47 como nómadas y 14 como visitantes, para un total de 105 especies.

Tabla I - Peces de la comunidad de residentes bénticos, indicando en cuales de las formaciones (A-F) fueron encontrados y su alimentación: $\mathrm{C}$. carnívoro; $\mathrm{P}$, planctófago; $\mathrm{O}$, Omnívoro y $\mathrm{H}$, herbívoro. Las especies marcadas? son secretivas, por ello no se asegura su presencia o ausen-
cia en esas formaciones.

Especie

Echidna catenata (Bloch)

Gymnothorax moringa (Cuvier)

G. vicinus (Castelnau)

Holocentrus ascensionis (Osbeck)

H. nufus (Walbaum)

Myripristis jacoous Cuvier

Scorpaena plumieri (Bloch)

Apogon maculatus (Poey)

Lutjanus mahogoni (Cuvier)

Haemulon boschmae (Metzelaar)

H. chrysargyreum Günther

H. flavolineatum (Desmarest)

$H$. melanurum (Linnaeus)

H. plumieri (Lacépede)

H. parrai (Desmarest)

$H$. sciurus (Shaw)

Equetus acuminatus (Bloch \& Schneider)

E. punctatus (Bloch \& Schneider)

Abudefduf saxatilis (Linnaeus)

Eupomacentrus dorsopunicans (Poey)

E. leucostictus (Müller \& Troschel)

E. partitus (Poey)

E. planifrons (Cuvier)

E. variabilis (Castelnau)

Microspathodon chrysurus (Cuvier)

Thalassoma bifasciatum (Bloch)

Sparisoma viride (Bonnaterre)

Opistognathus maxillosus Poey 1

$O$. whitehursti (Longley) 1

Ophioblennius atlanticus (Valenciennes)

Parablennius marmoreus (Poey) ${ }^{2}$

Coralliozetus cardonae Evermann \& Marsh

Labrisomus guppyi (Norman)

L. nigricinctus Rivero

L. nuchipinnis (Quoy \& Gaimard)

Malacoctenus triangulatus Springer

Starksia sp.

$S$, ocellata (Steindachner)

Bathygobius soporator (Valenciennes) 3

Coryphopterus dicrus (Böhlke \& Robins)

C. glaucofraenum Gill

Elacatinus illecebrosum (Böhlke \& Robins)

Gnatholepis thompsoni Jordan

Gobiosoma sp.

Acanthurus coeruleus Bloch \& Schneider

1. Construyen cuevas en el sedimento

Total

2. Habitan cabezas de Diploria

3. Encontrado al Sur del manglar

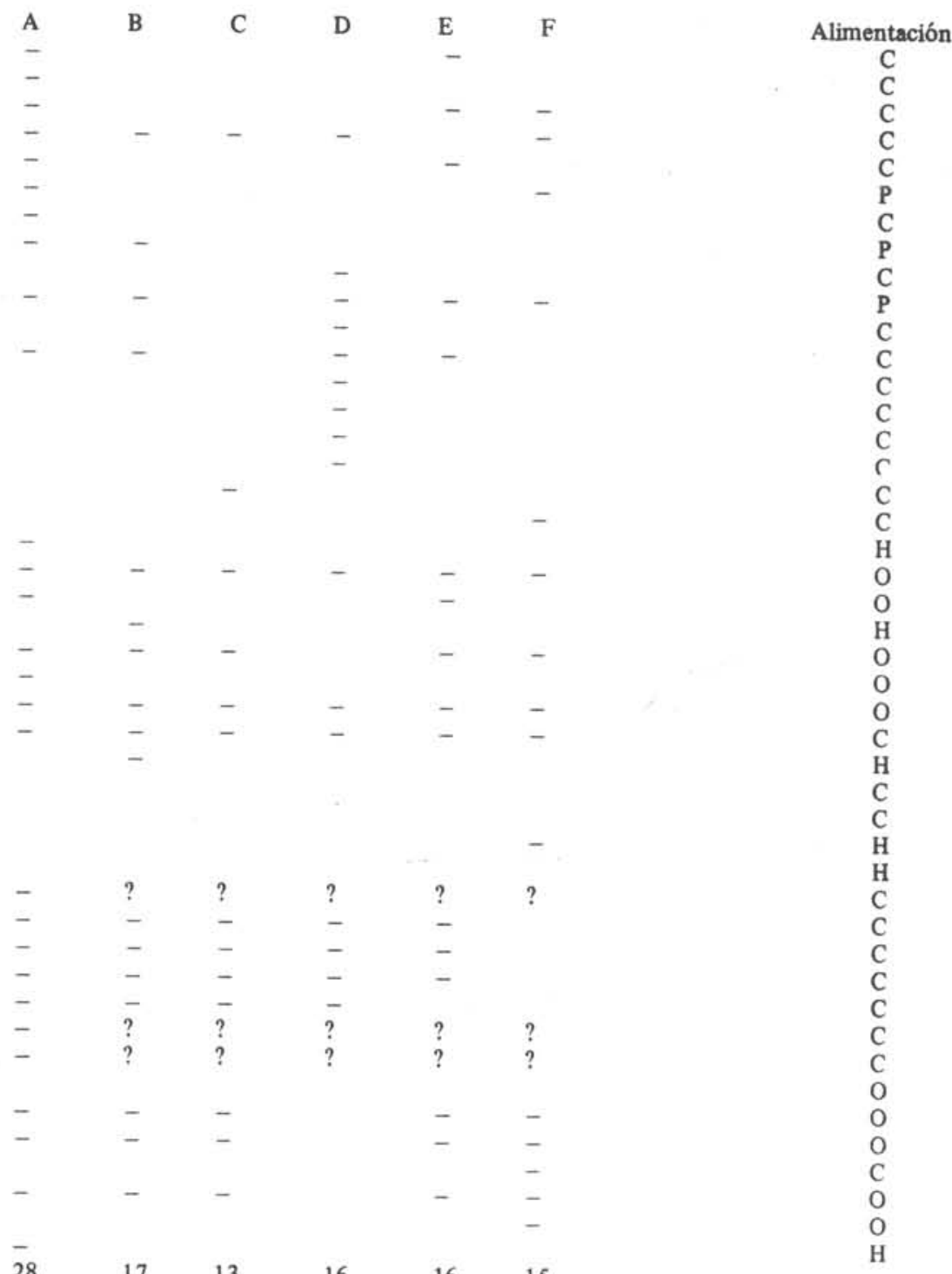

Tabla II - Peces de la comunidad de nómadas suprabénticos indicando su alimentación.

Especie

Myrichthys oculotus (Kaup)

Synodus intermedius (Agassiz)

Aulostomus maculatus Valenciennes

Syngnathus brachicephalus Poey

Dactylopterus volitans (Linnaeus)

Hypoplectrus puella (Cuvier)

Epinephelus striatus (Bloch)

Mycteroperca bonaci (Poey)

Caranx ruber (Bloch)

Lutianus analis (Valenciennes)

$L$ apodus (Walbaum)

L. griseus (Linnaeus)

L. synagris (Linnaeus)

Ocyurus chrysurus (Bloch)

Eucinostomus sp.

Alimentación
C
C
C
C
C
C
C
C
C
C
C
C
C
C
C

Especie

Gerres cinereus (Walbaum)

Anisotremus virginicus (Linnaeus)

Haemulon aurolineatum Cuvier

Calamus sp.

Odontoscion dentex (Cuvier)

Mulloidichthys martinicus (Cuvier)

Pseudupeneus maculatus (Bloch)

Pempheris schomburgki (Müller \& Troschel

Pomacanthus paru (Bloch)

Chaetodon capistratus Linnaeus

C. ocellatus Bloch

C. sedentarius Poey

$C$ striatus Linnaeus

Bodianus rufus (Linnaeus)

Halichoeres bivittatus (Bloch)
Alimentacio

C
C
C
C
C
C
C
P
O
C
C
C
C
C
C 
Especie

H. maculipinna (Müıler \& Troschel)

H. poeyi (Steindachner)

$H$. radiatus (Linnaeus)

Scarus croicensis Bloch

Sparisoma chrysopterum (Bloch \& Schneider)

$S$. radians (Valenciennes)

$S$. rubripinne (Valenciennes)

Acanthurus bahianus Castelnau

A. chirurgus (Bloch)

Cantherines pullus (Ranzani)

Monacanthus ciliatus (Mitchill)

Acanthostracion quadricornis (Linnaeus)

Lactopnrys bicaudalis (Linnaeus)

L. trigonus (Linnaeus)

L. triqueter (Linnaeus)

Canthigaster rostrata (Bloch)

Sphoeroides splengeri (Bloch)

Tabla III - Peces de la comunidad de visitantes de media agua y su alimentación.$$
\text { Especi }
$$

Jenkinsia sp.

Hemiramphus brasiliensis (Linnaeus)

Strongvlura notata (Poey)

Tylosurus sp.

Atherinomorus stipes (Müller \& Troschel)

Caranx hippos (Linnaeus)

C. latus Agassiz

Oligoplites saurus (Bloch \&Schneider)

Trachinotus goodei Jordan \& Evermann

Mugil curema Valenciennes

$M$. incilis Hancock

Sphyraena barracuda (Walbaum)

Ariomma regulus (Poey)

Alimentación
P
P
O
C
C
P
C
C
C
C
H
H
C
P

A1 comparar estos aatos con los encontrados por Smith \& Tyler $(1972,1973)$ (Tab. IV), se observa que ellos trabajaron en sitios donde la mayoría de los peces pertenecen a la comunidad de residentes, en tanto que en nuestra zona esta comunidad es desplazada por la de los nómadas. La comunidad de los visitantes permanece relativamente estable, siendo siempre la menos diversa en cualquier zona arrecifal.

Este desplazamiento de las comunidades se debe probablemente a que en la pradera el alimento es mas abundante que en zonas estrictamente coralinas, donde lo preponderante son las oportunidades de encontrar refugio. En la pradera, las formaciones coralinas son pequeñas otorgando una menor posibilidad de protección. Entonces, el desplazamiento de refugio a alimentación, favorece la abarición de peces que llegan a alimentarse en la zona, remplazando en abundancia a los residentes.

Tabla IV - Número de especies y porcentajes por comunidades encontrados en las Islas Virgenes (Smith \& Tyler, 1972), en las Bahamas (Smith \& Tyler,1973) y en Nenguange.

\begin{tabular}{|c|c|c|c|c|}
\hline & Residentes & Nómadas & Visitantes & Total \\
\hline Islas Virgenes & $53(73 \%)$ & & $27 \%)$ & 73 \\
\hline Bahamas & $51(57 \%)$ & $33(37 \%)$ & $5(6 \%)$ & 89 \\
\hline Colombia & $44(42 \%)$ & 47 (45\%) & $14(13 \%)$ & 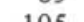 \\
\hline
\end{tabular}

\section{Relaciones con los Corales}

Al comparar las especies que se pueden encontrar en cada una de las formaciones de la pradera (Tab. V), se observa que existe una relación alta, mayor del $50 \%$, entre A, B, C y E, situadas al sur donde hay poco movimiento de agua y cierta sedimen-

Tabla V - Porcentajes de especies comunes entre las formaciones estudiadas.

$\begin{array}{ccccccc}\text { Formación } & \text { A } & \text { B } & \text { C } & \text { D } & \text { E } & \text { F } \\ \text { A } & - & 50 & 41 & 29 & 57 & 41 \\ \text { B } & 50 & - & 67 & 44 & 57 & 39 \\ \text { C } & 41 & 67 & - & 38 & 53 & 47 \\ \text { D } & 29 & 44 & 38 & - & 33 & 35 \\ \text { E } & 57 & 57 & 53 & 33 & - & 41 \\ \text { F } & 41 & 39 & 47 & 35 & 41 & -\end{array}$

tación, mientras que $D$ y $F$ situadas al norte, no sun afines en forma marcada con ninguna de las formaciones escogidas.

Se observa que la formación mas diversa es $A$, pues tiene $28(64 \%)$ de las especies residentes, en tanto que la que le sigue (B) solo tiene 17 (39\%). Quizas Millepora brinda una protección mas adecuada a los peces, lo que hace que invadan las formaciones donde este coral abunda. El otro extremo to tiene $A$. cervicor nis cuya formación (C) solo tiene $13(30 \%)$ de las especies residentes. Ya que la mayoría de los peces residentes de las formaciones de la pradera son pequeños, se puede pensar que los estrechos escondites de Millepora son los mas adecuados para ellos.

\section{Relaciones por Alimentacion}

Basandose en Randall (1967) se ubican los peces de la pradera en una categoría alimenticia, carnívoros, herbívoros, omnívoros y planctófagos. En la Tabla VI los datos alimenticios de las especies de cada comunidad se han convertido a porcentajes.

Tabla VI - Repartición de las comunidades ícticas en categorias alimenticias

$\begin{array}{ccccc} & \text { Carnívoros } & \text { Herbívoros } & \text { Omnívoros } & \text { Planctófagos } \\ \text { Residentes } & 26(58 \%) & 6(13 \%) & 10(22 \%) & 3\end{array}$

Carnívoros Herbívoros Omnívoros Planctófagos

$\begin{array}{lllll}\text { Residentes } & 26(58 \%) & 6(13 \%) & 10(22 \%) & 3(7 \%) \\ \text { Nómadas } & 37(79 \%) & 6(13 \%) & 3(6 \%) & 1(2 \%)\end{array}$

$\begin{array}{lllll}\text { Visitantes } & 7(50 \%) & 2(14 \%) & 1(7 \%) & 4(29 \%)\end{array}$

De esta manera se ve que la comunidad de peces residentes está integrada por carnívoros y omnívoros. Estos son los pomacnétridos, tratados aparte (Acero, 1978). Los peces carnivoros de esta comunidad, murenidos, holocéntridos y pomadásidos, son peces que realizan cortas migraciones para buscar alimento en la pradera, lo que hace que en ciertas horas no se les encuentre en su formación. Una especialización de los carnívoros residentes es la simbiosis de limpieza, utilizada por Elacatinus y Thalassoma.

Los nómadas se reparten en dos categorías, herbívoros y carnívoros. Los primeros son solo seis especies, scáridos y acantúridos, pero forman grandes cardúmenes que se mueven por la pradera, siendo mayoría en número de individuos. Los carnívoros son muy diversificados, pues los grandes predadores tienden a ser solitarios y acechan (Aulostomus) o se camuflan (Bothus, Synodus), en tanto que aquellos que comen invertebrados bentónicos, múllidos, chaetodóntidos y lábridos, desarrollan mecanismos especiales para extraerlos del sedimento y se mezclan con los herbívoros, aprovechando la protección que representan sus cardúmenes y la oportunidad de capturar la infauna levantada por estos al agitar la vegetación.

Los peces visitantes son principalmente planctófagos y carnívoros. Los primeros, clupeóideos y aterínidos, buscan refugio en la región protegida formada por los arrecifes, introduciendo energia cuando sirven de alimento a los peces carnívoros residentes o nómadas. Los carnívoros visitantes son peces que por los general se alimentan en grupos definidos, carángidos, bien arriba en la columna de agua, lo cual hace que su alimentación sean los peces planctófagos de su misma comunidad y ellos, debido a su tamaño relativamente grande, raramente sirven de alimento a los peces de otras comunidades. La excepción es Sphyraena, típico predador de las comunidades arrecifales.

\section{Conclusiones}

En la pradera estudiada la comunidad mas diversa es la de los nómadas suprabénticos, con el $45 \%$ de las especies, desplazando a los residentes bénticos (42\%). Esto se debe a que en la pradera el alimento es un factor mas caracteristico que la protección que brindan los corales. Los visitantes son el $13 \%$ de las especies.

La pradera tiene dos partes definidas, al sur donde las aguas son calmadas y con cierta sedimentación se forma un conjunto estable, como se ve al comparar los residentes de las formaciones situadas allí. Al norte las condiciones son mas agitadas con poca sedimentación, creandose un ambiente diferente. Millepora compla. nata es el coral utilizado por un mayor número de residentes como hábitat.

Los residentes son carnívoros, abandonando su refugio para alimentarse, excepto los pomacéntridos que son omnívoros. Los nómadas son herbívoros y carnívoros, los primeros son mayoría en número de individuos, pero son solo seis especies, el segundo grupo es mas diverso y se alimenta generalmente de invertebrados. Los visitantes se alimentan de plancton o de los mismos peces de su comunidad. 


\section{Agradecimientos}

Este trabajo forma parte de una tesis de grado para obtener el título de Biólogo Marino de la Facultad de Ciencias del Mar, Universidad de Bogotá Jorge Tadeo Lozano. Parte del patrocinio para su realización y exposición provino de COLCIENCIAS. El Dr. F. Köster (INVEMAR) contribuyó con la dirección y correción del original.

\section{Referencias Bibliográficas}

ACERO, P., A. 1978. Anotaciones ecólogicas y sistemáticas sobre los peces de la familia Pomacentridae en el Caribe colombiano. An. Inst. Inv. Mar. Punta de Bentín, $7(10)$.

BÖHLKE, J. E. \& CHAPLIN, C. C. G. 1968. Fishes of the Bahamas and adjacent tropicals waters. Wynnewood, $\mathrm{Pa}$., $\mathrm{Li}$ vingston, $771 \mathrm{p}$.
CAYCEDO, I. E. 1977. Fitoplancton de ta Bahia de Nenguange (Parque Nacional Tayrona), Mar Caribe, Colombia. An. Inst. Inv. Mar. Punta de Betín, (9):17-44.

CHAPLIN, C. C. G. \& SCOTT, P. 1972. Fishwatchers guide to West Atlantic coral reefs. Valley Forge, Pa., Harrowood, $64 \mathrm{p}$.

EHRLICH, P. R. 1975. The population biology of coral reef fishes. Annual Review Ecol. Syst., 6:211-47.

RANDALL, J. E. 1967. Food habits of reef fishes of the West Indies. Stud. Trop. Ocean., (5) : 060-847.

RANDALL, J. E. 1968. Caribbean reef fishes. Hong Kong, T. F.H., $318 \mathrm{p}$.

SMITH, C. L. \& TYLER, J. C. 1972 . Space resource sharing in a coral reef fish community. Nat. Hist. Mus. Los Angeles County, Sci. Bull., 14:125-70.

SMITH, C. L. \& TYLER, J. C. 1973. Population ecology of a Bahamian suprabenthic shore fish assemblage. Am. Mus. Novitates, (2528):1-38. 